\title{
Affective Forecasting and Self-Control: Why Anticipating Pride Wins Over Anticipating Shame in a Self-Regulation Context
}

\author{
Vanessa M. Patrick, University of Houston \\ HaeEun Helen Chun, University of Southern California \\ Deborah J. Macinnis, University of Southern California
}

\begin{abstract}
We demonstrate that anticipating pride from resisting temptation facilitates selfcontrol due to an enhanced focus on the self while anticipating shame from giving in to temptation results in self-control failure due to a focus on the tempting stimulus. In two studies we demonstrate the effects of anticipating pride (vs. shame) on self-control thoughts and behavior over time (Studies 1 and 2) and illustrate the process mechanism of self vs. stimulus focus underlying the differential influence of these emotions on self-control (Study 2). We present thought protocols, behavioral data (quantity consumed) and observational data (number/size of bites) to support our hypotheses.
\end{abstract}

Consumers often anticipate how their choices will make them feel. Anticipating feelings from consumption is important because anticipated emotions can help to clarify our preferences (Kahneman \& Snell, 1992) and influence the choices we make (Gilbert et al., 1998). Despite their relevance to consumer choice, the impact of anticipated emotions on emotional and behavioral regulation, especially in the domain of self-control is under-studied (Baumeister et al., 2007, 2008), though posited to be quite powerful (Maclnnis \& Patrick, 2006).

More than two thousand years ago Socrates asserted in Plato's Phaedrus (approximately 370 $\mathrm{BC}$ ) that there are two horses in the soul, the one-unruly, governed by passions and constantly pulling in the direction of pleasure; the other restrained, dutiful, obedient and governed by a sense of shame. Shame can be a powerful regulator of human behavior (Tangney \& Dearing, 2002). However, when faced with a tempting stimulus, one might anticipate not only shame from succumbing to the temptation, as proposed by Socrates, but also pride from not succumbing and resisting temptation (c.f. Mukhopadhyay and Johar, 2007). Recent research uncovers the beneficial effects of pride in a variety of domains (Tangney, 1999; Tracy \& Robins, 2004, 2007). However, the role of anticipated pride (and 
shame) on self-control has not been investigated and hence serves as a focal point of the current research.

We know little about whether, and to what extent, anticipating emotions impacts self-control. Thus, this research contributes to the literature in several key ways. We develop a theoretical framework that explains how anticipating emotions of pride and shame facilitates vs. undermines selfcontrol efforts and why. We hypothesize and find that anticipating pride from resisting temptation enhances self-control while anticipating shame from giving in to temptation does not. We also demonstrate the process mechanism underlying this effect, suggesting that the differential effect of anticipated pride vs. shame on self-control is tied to the attentional focus each anticipated emotion evokes. Anticipated shame focuses on the stimulus and its consummatory properties while anticipated pride focuses attention on the self and the goal of self-control. Finally, since self-control efforts can be psychologically taxing (Baumeister et al., 2007, 2008; Vohs, 2006), we investigate whether the impact of anticipated emotions on self-control varies as a function of the amount of time consumers are exposed to a tempting stimulus.

These issues are addressed in two studies. Study 1 illustrates that anticipating pride (but not shame) facilitates self-control. Specifically, consumers who anticipate pride from resisting temptation (a) consume less, (b) have fewer thoughts about the stimulus itself, (c) have more self-control thoughts, and (d) engage in more controlled and strategic consumption over time. We theorize that anticipating pride enhances self-control because it diverts attention from the stimulus and consumption and instead focuses attention on the self. Study 2 tests this process mechanism by manipulating both anticipated emotions (pride vs. shame) and self-focus (high vs. low). We show that increasing self-focus in the anticipated shame condition enhances self-control but has no additional impact on self-control in the anticipated pride condition.

\section{Theoretical Framework and Hypothesis}

\section{Anticipated Emotions and Self-Control}

Anticipated emotions, also called affective forecasts, are predictions of the emotional consequences of decision outcomes (Bagozzi, Baumgartner, \& Pieters, 1998; Gilbert et al., 1998; Maclnnis, Patrick, \& Park, 2005), in this case the consequences of giving in or not giving in to temptation. Thus, we may ask ourselves questions such as: "How would I feel if I chose to eat (or not eat) that piece of chocolate cake?"; "How will I feel if I steal (resist stealing) right now?" Although our choices may not 
always make us feel the way we anticipated they would (Patrick, Maclnnis \& Park, 2007), emotions anticipated to arise from choices do help us order our preferences and are often critical inputs to the choices we make (Kahneman \& Snell, 1992).

Prior research is suggestive of the impact of anticipated emotions on self-control. A set of studies by Bagozzi et al. demonstrated that anticipated positive and negative emotions predict selfcontrol intentions but anticipated positive emotions, in general, have a more positive impact on selfcontrol intentions than do anticipated negative emotions (Bagozzi et al., 1998). Notably though, limited research has examined the impact of specific positive and negative anticipated emotions on self-control outcomes. We contribute to the literature by suggesting that the effect of anticipated emotions on undermining or facilitating self-control depends on the properties of the specific anticipated emotion (see Fig. 1).

When faced with a tempting stimulus, shame may be anticipated if one succumbs to temptation (Tangney \& Dearing, 2002) whereas pride may be anticipated if one resists temptation (Mascolo \& Fischer, 1995). Interestingly, little consumer research has examined the impact of specific anticipated emotions on enhancing self-control. A notable exception is Mukhopadhyay and Johar (2007) who contrast the effects of happiness (pleasure) and pride on buying vs. not-buying when presented with an unintended purchase opportunity. Notably though, they focus on the impact of experienced emotions, not anticipated emotions, on buyer behavior. Nor do they contrast pride with shame.

Although several other emotions like pleasure, deprivation and guilt may be anticipated in a self-regulation context, the unique similarities and differences in anticipated pride and shame make comparisons between these emotions particularly interesting. Anticipated pride and anticipated shame are similar in their potential to facilitate self-control (Giner-Sorolla, 2001). In contrast, anticipating emotions such as the pleasure from consumption or the deprivation from non-consumption seems more relevant to undermining (not fostering) self-control. Anticipating guilt has been contrasted with anticipating shame in previous research (Chun, Patrick, \& Maclnnis, 2006) and has also been found to undermine self-control compared to anticipated shame, thus a focus on anticipating shame provides a more conservative test.

Despite their similarity in facilitating self-control, the emotions of pride and shame differ in attentional focus and valence; factors that are critical to the manner in which they impact self-control. Specifically, anticipating pride is positively valenced and focuses attention on the self, whereas anticipating shame is negatively valenced and focuses attention on the tempting stimulus. Since the manner in which these emotions operate is different, we expect that they also vary in the extent and 
reliability with which they facilitate self-control. Thus, our central thesis is that the impact of anticipated shame and pride on self-control is attributed to the inherent properties of these two emotions and the underlying process by which they operate. Research on delay of gratification and emotion regulation provides support for our predictions. Hence, we briefly review that literature next.

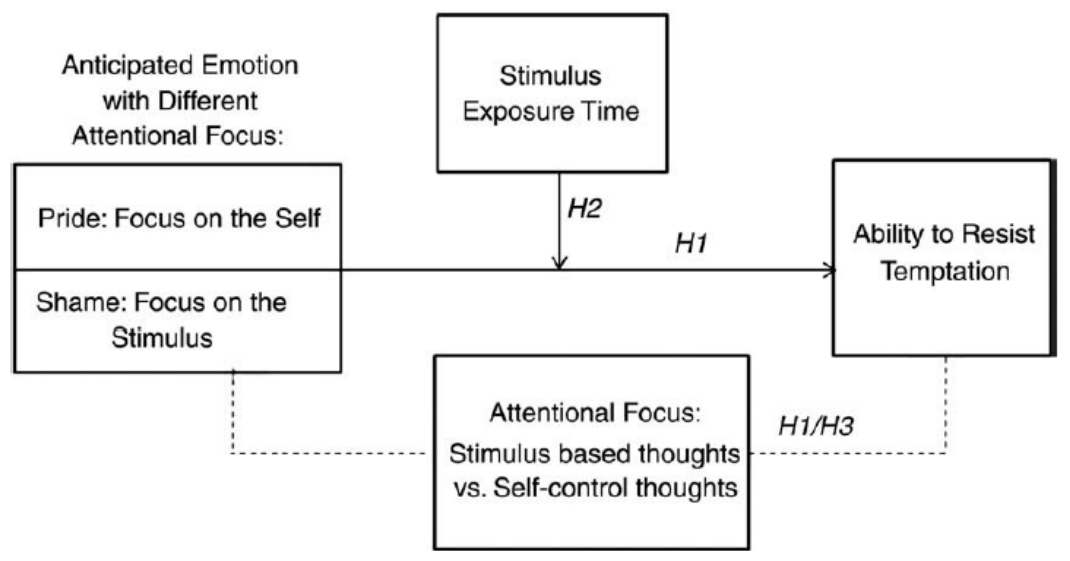

Fig. 1. Conceptual model.

\section{Hot and Cold System for Resisting Temptation}

Succumbing to or resisting temptation is governed by two interacting systems: the "hot" and the "cool" system (Metcalfe \& Mischel, 1999). The hot system is activated by a tempting stimulus that has gained emotion-laden associations through past experiences. Activation of the hot system creates a reflexive, rapid "go" response, reducing one's capacity to resist a tempting stimulus. Hence, activation of the hot system induces a motivation to succumb to temptation.

Various strategies can, however, be implemented to make the stimulus less "hot." Such strategies are facilitated via a second "cool" system, driven by cognition, not emotions (see also Strack, Werth, \& Deutsch, 2006; Vohs, 2006). This system is complex, slow and reflective, and focuses on "know" responses that involve cognitively-based strategic efforts to resist the tempting stimulus. Activation of the cool vs. hot system is impacted by one's attentional focus. Attention appears to modulate the draw of the hot system. For example, research shows that self-control can be enhanced when individuals focus on the non-consummatory aspects of the stimulus, such as the relationship of the tempting stimulus to other objects and activities (e.g., Mischel \& Baker, 1975). Temptation can also be reduced by paying attention to other stimuli that are not connected to the "go" response. Thus, distracting attention away from the tempting stimulus to focus on other stimuli can reduce the desire to succumb to the tempting stimulus (Mischel, Ebbesen, \& Zeiss, 1972). Indeed, prior research has shown 
that attention to the self increases inhibitions in response to indulgence or cheating (Heatherton et al., 1993). We build on these findings to argue that the self-focus activated by anticipating pride shifts attention from the stimulus to the self and thus hinders the activation of the hot system and rapid "go" response.

\section{Anticipated Emotions and the Hot-Cool System}

Without any instructions to anticipate a specific emotion, we expect that the default response on exposure to a tempting stimulus will be to succumb to temptation based on the view that the stimulus activates the hot system, reducing one's capacity to resist temptation. Would anticipating shame from consumption reduce the activation of the hot system, resulting in more self-control compared to this default condition? Shame has been conceptualized as a negative emotion arising from attention to and evaluations of negative actions (Lewis, 1971; Tangney \& Dearing, 2002). Shame has been shown to have strong links with depression, perhaps because it is perceived to be caused by a personal failure or moral transgression (Ortony, Clore, \& Collins, 1988). We expect that anticipating shame from consuming a tempting stimulus focuses attention on the stimulus and thus emphasizes the consequences of giving in to temptation and consumption. We hypothesize that due to this attentional focus, anticipating shame retains a focus on the "hot" stimulus and thus does not have the capacity to shift attention from this hot stimulus to the self or other stimuli that are not connected to the "go" response.

In contrast, pride has been conceptualized as a positive emotion arising from achievements that are attributed to one's abilities and efforts (Lewis, 1971; Tangney, 1999; Tracy \& Robins, 2004; 2007). In previous research, pride has been shown to be particularly efficacious as a motivator, providing an incentive to pursue longer-term goals despite short-term losses. Thus we expect that anticipated pride facilitates activation of the cool system by distracting attention from the consummatory or "hot" properties of the stimulus to the self and longer-term effects to the self from non-consumption. Our reasoning is consistent with prior research, which shows that self-control is enhanced when attention is distracted from the consummatory properties of the stimulus (Mischel et al., 1972; Mischel \& Baker, 1975). This leads us to predict that:

Hypothesis 1: When consumers are exposed to a tempting stimulus, anticipating pride associated with resisting temptation facilitates self-control thoughts and behaviors relative to anticipating shame associated with giving in to temptation as well as a no anticipated emotions control condition. Anticipating shame will be no more efficacious than a no anticipated emotion control. 
How Long-Lasting is the Influence of Anticipating Pride on Self-Control?

Resisting temptation is an effortful process that can tax one's self-regulatory capacities over time (Baumeister et al., 1998, 2008; Vohs, 2006). The effortful nature of this process should be more pronounced when the temptation continues over an extended period of time (e.g., when a piece of cake is placed next to one's plate during an extended meal). Hypothesis 1 suggests that anticipating pride facilitates self-control thoughts and behaviors. However, a more interesting question concerns how efficacious this strategy is over time with continuous exposure to the hedonic stimulus. In other words, would the facilitative impact of anticipated pride hypothesized above be weakened or sustained over time? Based on our theorizing above, we expect that although the effects of anticipated pride might diminish over time, self-control lapses will be strategic, controlled, and monitored, thereby resulting in persistent self-control capacities. Thus, while the pull of the hot system may strengthen over time, the attentional focus on the self allows consumers to keep the ultimate goal (feeling pride) in mind. Rather than completely succumbing to temptation, consumers may give in to temptation in a small way (e.g., having a little taste; having a few tastes over time but portioning them out over time), but still retain a focus on the positive benefits of restraint. See Mukhopadhyay and Johar (2009). Thus,

Hypothesis 2: When consumers are faced with a tempting stimulus over time, anticipating pride associated with resisting temptation has a positive impact on self-control compared to anticipating shame associated with giving in to temptation as well as a no anticipated emotions control condition over both the short- and long-term.

We test Hypotheses 1 and 2 in Study 1.

\section{Study 1}

\section{Design and Procedure}

Ninety five students and staff at a large University were paid \$15 to participate in a study on "food products and consumption." Respondents were invited to the laboratory and were randomly assigned to one of a set of rooms equipped with a video camera. Respondents were asked to sit at a desk on which was placed a covered container housing a large piece of rich chocolate cake from the Cheesecake Factory. The container's clear plastic top enabled respondents to readily view the cake. A bottle of water, a fork, and napkins sealed in a plastic packet, a sheet of paper, a pencil, and a timer were also on the desk. 
Respondents were asked to open the container and examine the cake. They were instructed to eat as much or as little of the cake as they desired, but also to anticipate how much shame they would feel as a result of eating the cake or how much pride they would feel as a result of not eating the cake. A control group was not given any instructions about anticipating emotions. Respondents were further instructed to record any thoughts that occurred to them while they sat in the room and to use the timer to note the time at which each thought occurred. Respondents were video recorded from the time they entered the room until the time they left. The experimenter privately weighed the leftover cake following the session. Additional data like time of day, extent of hunger, individual differences in liking for chocolate cake, and whether consumers were on a diet were also collected. We found no differences in these variables across the experimental conditions. The results did not differ when these variables were included as covariates in the analyses. Hence, they are not discussed further.

Trained coders blind to the hypotheses and experimental condition viewed the videos and coded respondents' behavior. They also coded the thought logs and categorized thoughts as (1) thoughts that focus on self-control and resisting temptation (e.g. "I don't need this cake", "other foods are better for me", and "I'm going to show restraint") and (2) thoughts that reflected a focus on the tempting stimulus (e.g. "The cake looks creamy", "It smells so good", and "It will taste great"). A thought protocol analysis was conducted to determine whether pride and shame had differential effects on consumers' attentional focus, that is whether they focus on the tempting stimulus or whether they focus on the self and the goal of self-control.

A comprehensive database that contained the coded behavioral data, coded thoughts, selfreport data, and the total amount of cake consumed was subsequently created. This database allowed for measures of the classes of variables relevant to Hypotheses 1 and 2.

\section{Measures}

\section{Self-Control Behavior}

Recent research in consumer psychology conceptualizes self-control not only in terms of choice but also in terms of consumption (Garg, Wansink, \& Inman, 2007; Fedorikhin \& Patrick, 2009).

Analogously, we test Hypothesis 1 by measuring self-control in terms of both whether and how much of the tempting stimulus is consumed. Specifically, we examined (1) whether respondents ate the cake at all, (2) how much they consumed (the difference between its pre- and post-session weight), (3) the number and size of bites they took, and (4) the weighted number of bites. Variables 3 and 4 were obtained from the video recording of the consumption episode. Coders recorded when during the 10 
minute period respondents took a bite. They also estimated the bite's size. A weighted measure of consumption was computed by multiplying the number of bites times the size of the bite (1=small; $2=$ medium; 3=large).

Self-Control Thoughts and Thoughts about the Tempting Stimulus

Coders blind to the respondent's condition read respondents' thought logs and listed the number of self-control thoughts as well as the number of thoughts that focused on the tempting stimulus.

\section{Exerting Self-Control over Time}

Self-control was measured by observing (1) the percentage of respondents who completely resisted the cake (i.e., did not eat any cake) in the first and second 5 min of the experimental session, (2) the total number and size of bites the individual took in the first and second $5 \mathrm{~min}$, and (3) the weighted number of bites in the first and second $5 \mathrm{~min}$ of the experimental session. The assumption here is that an increase in bite number and size over time indicates diminished self-control ability. We also developed a visual trend by counting the frequency of bites in 30 second intervals during the 10 minute period. Finally, thought data provided insight into potential changes in self-control over time.

\section{$\underline{\text { Results }}$}

\section{Self-Control Behavior}

Respondents in the anticipated pride condition were more likely to completely resist the cake (40\%) compared to respondents in the control condition (18.8\%) and those in the anticipated shame condition (10.5\%). These differences were only directionally significant however ( $z=1.37$ and 1.74 for the control and anticipated shame conditions, respectively, $p>.05$, for both tests), perhaps because of the small sample size. As hypothesized, there were no differences between the control condition and the anticipated shame condition in the percentage of respondents who completely resisted the cake. See Table 1.

Those in the anticipated pride condition who consumed the cake ate significantly less $\left(M_{\text {pride }}=1.23 \mathrm{oz}\right)$ than did those in the control condition $\left(M_{\text {control }}=3.08 \mathrm{oz} ; \mathrm{t}=2.18, p<.05\right)$. They also ate less than did those in the anticipated shame condition $\left(\mathrm{M}_{\text {shame }}=2.63 \mathrm{oz} ; \mathrm{t}=-2.53, \mathrm{p}<.05\right)$. They also took fewer bites of the cake than both those in the control condition $\left(M_{\text {pride }}=5.35\right.$ vs. $\left.M_{\text {control }}=13.25 ; t=2.40, p<.05\right)$ and those in the anticipated shame condition $\left(\mathrm{M}_{\text {shame }}=11.32 ; \mathrm{t}=2.30, \mathrm{p}<.05\right)$. Respondents in the anticipated pride condition also ate the cake in a more controlled manner as revealed by the 
significantly lower number of weighted bites (number of bites times bite size) than those in the control condition $\left(M_{\text {pride }}=9.75\right.$ vs. $\left.M_{\text {control }}=26.0, t=2.51, p<.05\right)$ and a marginally lower number of bites than those in the anticipated shame condition $\left(\mathrm{M}_{\text {shame }}=18.05 ; \mathrm{t}=1.88, \mathrm{p}=.07\right)$. The anticipated shame condition did not significantly differ from the control condition on any of these variables. These results support Hypothesis 1.

\section{Self-Control Thoughts and Thoughts about the Tempting Stimulus}

An analysis of the thought protocols supports the theorizing underlying Hypothesis 1 .

Respondents in the anticipated pride condition had significantly more self-control thoughts $\left(\mathrm{M}_{\text {pride }}=\right.$ 3.95) compared to those in the control $\left(\mathrm{M}_{\text {control }}=.53 ; \mathrm{t}=-4.90, \mathrm{P}<.05\right)$ and the anticipated shame conditions $\left(\mathrm{M}_{\text {shame }}=1.58 ; \mathrm{t}=-2.76, \mathrm{p}<.05\right)$. The anticipated shame condition did not differ from the control condition in terms of the number of self-control thoughts. Respondents in the anticipated pride condition also had significantly fewer thoughts about the tempting stimulus ( $M_{\text {pride }}=3.57$ ) compared to consumers in the control condition $\left(\mathrm{M}_{\text {control }}=5.71 ; \mathrm{t}=2.42, \mathrm{p}<.05\right)$ and those in the anticipated shame condition $\left(\mathrm{M}_{\text {shame }}=6.42 ; \mathrm{t}=3.31, \mathrm{p}<.05\right)$. The anticipated shame condition did not differ from the control condition in terms of the number of thoughts about the tempting stimulus. These results also support Hypothesis 1.

Table 1

Study 1: the effect of anticipated emotions on the ability to resist temptation.

\begin{tabular}{|c|c|c|c|}
\hline & Anticipated pride & Anticipated shame & No emotion control \\
\hline \multicolumn{4}{|l|}{ Self-control success: ability to resist temptation $(\mathrm{HI})$} \\
\hline$\%$ completely resisting the cake & $40.0 \%$ & $10.5 \%$ & $18.8 \%$ \\
\hline Total amount consumed in oz & $1.23^{\mathrm{a}}$ & $2.63^{\mathrm{b}}$ & $3.08^{\mathrm{b}}$ \\
\hline Total number of bites & $5.35^{\mathrm{a}}$ & $11.32^{\mathrm{b}}$ & $13.25^{\mathrm{b}}$ \\
\hline \multicolumn{4}{|l|}{ Attentional focus (H3) } \\
\hline No. of thoughts about the tempting stimulus & $3.57^{\mathrm{a}}$ & $6.42^{\mathrm{b}}$ & $5.71^{\mathrm{b}}$ \\
\hline \multicolumn{4}{|l|}{ Resisting temptation over time (H2) } \\
\hline$\%$ completely resisting the cake in first $5 \mathrm{~min}$ & $55.0 \%{ }^{\mathrm{ac}}$ & $15.8 \% \%^{\mathrm{b}}$ & $25.0 \%{ }^{\mathrm{ab}}$ \\
\hline$\%$ completely resisting the cake in last $5 \mathrm{~min}$ & $40.0 \%{ }^{\mathrm{c}}$ & $10.5 \%$ & $18.8 \%$ \\
\hline Weighted bites in first $5 \mathrm{~min}$ & $3.65^{\mathrm{ac}}$ & $10.47^{\mathrm{b}}$ & $13.25^{\mathrm{b}}$ \\
\hline Weighted bites in last $5 \mathrm{~min}$ & $6.10^{\mathrm{a} * \mathrm{c}}$ & $7.58^{\mathrm{ab}}$ & $12.75^{\mathrm{b}^{*}}$ \\
\hline Number of bites in first $5 \mathrm{~min}$ & $1.80^{\mathrm{ac}}$ & $6.11^{\mathrm{b}}$ & $6.56^{\mathrm{b}}$ \\
\hline Number of bites in last $5 \mathrm{~min}$ & $3.55^{\mathrm{c}}$ & 5.21 & 6.69 \\
\hline Small bites in last $5 \mathrm{~min}$ & $1.45^{\mathrm{c}}$ & 2.53 & 2.00 \\
\hline Medium bites in first $5 \mathrm{~min}$ & 1.35 & $1.26^{\mathrm{c}}$ & 2.94 \\
\hline Medium bites in last $5 \mathrm{~min}$ & $0.85^{\mathrm{a}}$ & $2.68^{\mathrm{bc}}$ & $2.69^{\mathrm{b}}$ \\
\hline Large bites in first $5 \mathrm{~min}$ & $0.50^{\mathrm{a}^{*}}$ & $0.84^{\mathrm{ab}}$ & $2.00^{\mathrm{b*}}$ \\
\hline Large bites in last $5 \mathrm{~min}$ & 0.65 & 0.84 & 1.63 \\
\hline No. of thoughts about the tempting stimulus in first $5 \mathrm{~min}$ & $2.48^{\mathrm{ac}}$ & $3.79^{\mathrm{b}}$ & $3.94^{\mathrm{bc}}$ \\
\hline No. of thoughts about the tempting stimulus in last $5 \mathrm{~min}$ & $1.10^{\mathrm{ac}}$ & $2.63^{\mathrm{b}}$ & $1.76^{\mathrm{abc}}$ \\
\hline No. of thoughts about resisting the tempting stimulus in first $5 \mathrm{~min}$ & $1.86^{\mathrm{a}}$ & $0.79^{\mathrm{b}}$ & $0.24^{\mathrm{b}}$ \\
\hline No. of thoughts about resisting the tempting stimulus in last $5 \mathrm{~min}$ & $2.10^{\mathrm{a}}$ & $0.79^{\mathrm{b}}$ & $0.29^{\mathrm{b}}$ \\
\hline
\end{tabular}

Superscripts $\mathrm{a}$ and $\mathrm{b}$ show significant differences between the conditions in the same row, superscripts $\mathrm{a}^{*}$ and $\mathrm{b}^{*}$ show marginally significant differences between the conditions in the same row, and superscript $\mathrm{c}$ show significant changes in the first 5 and last $5 \mathrm{~min}$. 


\section{Exerting Self-Control over Time}

Table 1 shows that self-control abilities of consumers in the anticipated pride condition tended to weaken over the duration of the consumption. However, consistent with Hypothesis 2, anticipating pride resulted in more self-control over the duration of the consumption episode, compared to anticipating shame and the no-emotion control. Fifty five percent of respondents in the anticipated pride condition were able to completely resist the cake in the first $5 \mathrm{~min}$ (compared to $15.8 \%$ for the anticipated shame condition; $z=2.22, p<.05$ and $25.0 \%$ for the control condition; $z=1.47, p=n s) ; 40 \%$ were still able to resist it in the second 5 min compared to $10.5 \%$ in the anticipated shame condition, although this difference is no longer significant. Moreover, although the number of bites increased between the two time intervals, respondents in the anticipated pride condition took fewer bites of the cake (regardless of bite size) than did respondents in the anticipated shame and control conditions. An analysis of the weighted bites (number of bites multiplied by size of bites) showed a significant increase in weighted bites from the first $(M=3.65)$ to the second 5 minute time interval $(M=6.10 ; t=-2.04, p<.05)$. Despite this decrease in restraint, weighted bites for respondents in the anticipated pride condition were significantly less than those for the control condition $\left(M_{\text {pride }}=3.65\right.$ vs. Mcontrol=13.25; $t=2.83$, $p<.05)$ and those in the anticipated shame condition $\left(M_{\text {shame }}=10.47 ; t=2.22, p<.05\right)$ in the first 5 min and

directionally fewer bites than those for the control condition in the second 5 minute period $\left(M_{\text {pride }}=6.10\right.$ vs. $M_{\text {control }}=12.75 ; t=1.99, p=.09$; see Table 1). The anticipated shame condition did not differ from the control condition over the first or the second 5 minute periods.

Next, we decoupled the weighted results described above to analyze the total number of bites and the size of the bites across the two 5 minute periods. An analysis of the total number of bites showed that respondents in the anticipated pride condition took more bites during the second $(M=3.55)$ vs. the first 5 minute period $(M=1.80 ; t=-2.36, p<.05)$. However, consistent with our theorizing, they took fewer bites than did respondents in the control and anticipated shame conditions. Fig. 2 shows a visual representation of the frequency of bites counted in 30 second intervals over the 10 minute period. Despite a slight increase in bites after the first $5 \mathrm{~min}$, the overall trend of the graph in the anticipated pride condition reveals a consistent effect of anticipated pride on self-control over time. An analysis of the size of the bites revealed that subjects in the anticipated pride condition took more small bites in the second $(M=1.45)$ vs. the first time period $(M=.45 ; t=-2.21, p<.05)$, but no change in the number of medium or large bites they took across the two periods. Thus while respondents in the anticipated pride condition took more bites in the second $5 \mathrm{~min}$, they did so by eating smaller bites, reflective of strategic control over consumption rather than a lapse of restraint. 
The verbal data also reflect consistent self-control effects in the anticipated pride condition over

time. Respondents in the anticipated pride condition sustained self-control thoughts and thoughts about the tempting stimulus to the same degree over time. Thus, compared to both the control and the anticipated shame conditions, they had significantly more self-control thoughts during both the first $\left(M_{\text {pride }}=1.86\right.$ vs. $\left.M_{\text {control }}=.24, t=-4.04, p<.05 ; M_{\text {shame }}=.79, t=-2.30, p<05\right)$ and second 5 minute periods $\left(M_{\text {pride }}=2.10\right.$ vs. $\left.M_{\text {control }}=.29, t=3.94, p<.05 ; M_{\text {shame }}=.79, t=-2.61, p<.05\right)$. Moreover, respondents who anticipated pride had significantly fewer thoughts about the tempting stimulus during the second $(M=1.10)$ vs. the first 5 minute period $(M=2.48 ; t=3.11, p .05)$. As predicted, there was no difference between respondents in the anticipated shame and control conditions in the number of these thoughts over time. Overall, our results support Hypothesis 2. Although a tempting stimulus can decrease selfcontrol over time, anticipating pride from resisting the stimulus has a significant impact on self-control both over the short- and long-term.

\section{$\underline{\text { Discussion }}$}

Study 1 used self-reported data (thought protocols), behavioral data (quantity consumed) and observational data (number and size of bites) to demonstrate that (a) anticipated pride results in enhanced self-control, (b) it has the greatest ability to shift attentional focus from the tempting stimulus to the self and to the goal of self-control, and (c) it results in persistent efforts towards self-control even upon a longer-term exposure to a tempting stimulus. We find that anticipated shame is not effective at enhancing self-control. Our results suggest that anticipated shame is not sufficiently strong to override the impulse generated by the "hot" properties of the stimulus and thus has less capacity to facilitate self-control.

We theorized that anticipating pride has a positive effect on self-control because this emotion redirects attention from the "hot" tempting stimulus to the "cool" self. In Study 2 we further investigate the facilitative impact of anticipated pride on self-control by attempting to illuminate this process mechanism. Study 1 provided evidence from the thought protocols supporting this attentional focus, showing that consumers who anticipated pride had more self-control thoughts and fewer thoughts about the tempting stimulus compared to consumers in the control and anticipated shame conditions. No differences in self-control thoughts or thoughts about the stimulus were observed for consumers in the anticipated shame and control conditions.

Study 2 explores this attentional focus process explanation more directly by manipulating attentional focus. If attentional focus is indeed responsible for the positive effects of anticipated pride 
relative to anticipated shame, we should find that a manipulation that focuses the attention of those who anticipate shame on the self as opposed to the consummatory properties of the stimulus facilitates self-control. Thus, consumers who are asked to anticipate shame and who focus on the self should show greater self-control than consumers who are asked to anticipate shame but who are not asked to focus on the self. In contrast, consumers who anticipate pride should show greater self-control regardless of whether they are explicitly asked to focus on the self or not, due to the inherent self-focus induced from anticipating pride.

Hypothesis 3: The facilitative effects of anticipated pride are attributed to its ability to shift attention from the tempting stimulus to the self. Thus, consumers in the anticipated shame condition should show as much self-control as consumers in the anticipated pride condition when their attention is distracted from the stimulus and focused on the self.

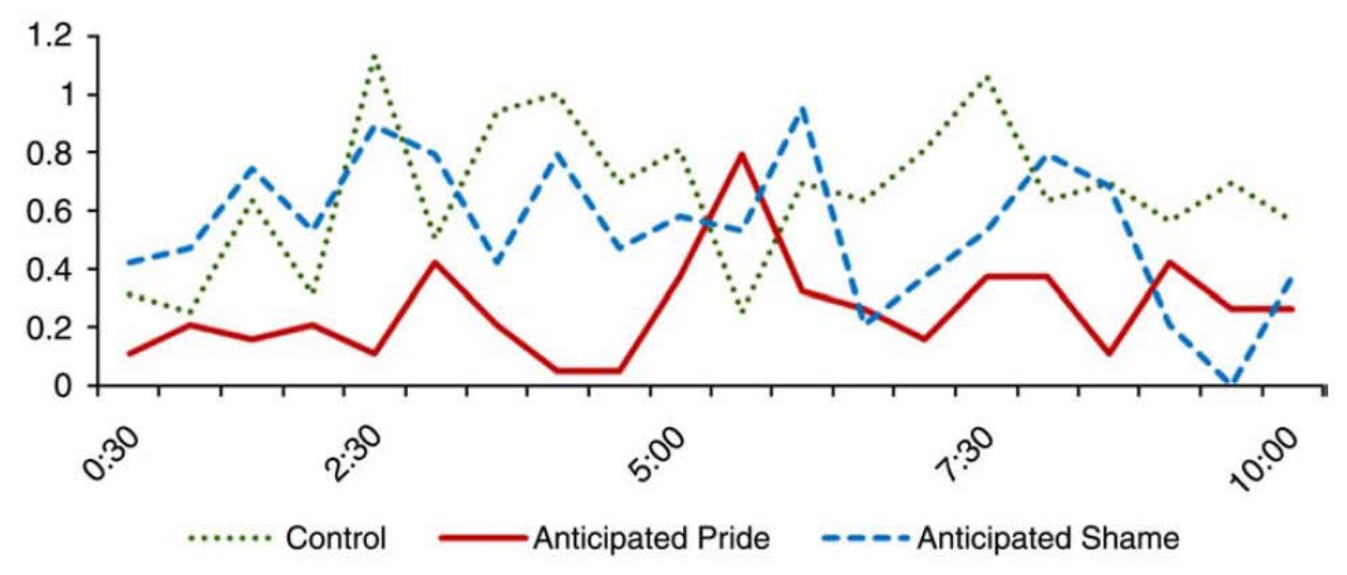

Fig. 2. Frequency of bites over time according to the anticipated emption conditions.

One might argue that an alternative explanation underlies the differential self-control efficacy of anticipated pride vs. shame. Recall that a prior work (Bagozzi et al., 1998) found that anticipated positive emotions had a more positive impact on self-control than did anticipated negative emotions. Hence, in contrast to $\mathrm{H} 3$, perhaps pride "works" because it emphasizes positive outcomes and good feelings, whereas shame emphasizes negative outcomes and bad feelings. Thus, perhaps anticipating shame is less effective as a self-control mechanism than anticipating pride not because it focuses attention on the tempting stimulus and its consummatory properties, but rather because it emphasizes negative outcomes that make people feel bad. Anticipated pride, by contrast, may facilitate self-control not because it refocuses attention from the tempting stimulus to the self, but because it emphasizes positive outcomes that make people feel good. However, if this alternative explanation were correct, 
merely shifting consumers' attention from the tempting stimulus to the self would not be sufficient to impact self-control for consumers who anticipate shame. Rather, anticipated shame should be less effective at inducing self-control even when attention shifts to the self. This is so because the negative feelings induced by anticipating shame are responsible for undermining self-control. This explanation would reveal a different pattern of results compared to those described in $\mathrm{H} 3$. Rather than finding an interaction between anticipated emotion and attentional focus (as suggested by $\mathrm{H} 3$ ), we should find that anticipated pride is more effective at inducing self-control regardless of attentional focus. We rule out this alternative explanation in the study that follows.

\section{Study 2}

Study 2 used a 2 (anticipated emotion: pride vs. shame)×2 (self-focus: high vs. low) between subjects experiment. Anticipated emotions were manipulated in a manner identical to Study 1 . Study 2 did not include a control condition primarily because the goal was to illustrate the process mechanism that underlies the differential influence of anticipating pride (vs. shame) on self-control, not to replicate the efficacy of anticipated pride and shame vis a vis a no anticipated emotions control group.

The presence of a mirror in which respondents could see their own reflection throughout the experiment served to manipulate attentional focus on the self. The presence of mirrors (or other selffocusing cues) has been shown to reduce the likelihood of cheating on a test (Diener \& Wallbom, 1976) and stealing Halloween candy (Beaman, Klentz, Diener, \& Svanum, 1979). Consistent with H3, we expect that if self-focus explains the facilitative effects of anticipated pride on self-control, consumers who anticipate pride from non-consumption and who are made to feel a heightened sense of the self by sitting in front of a mirror will act no differently from consumers who do not sit in front of a mirror. This is so since anticipated pride already directs attention to the self, rendering the self-focus manipulation ineffective at changing the impact of anticipated pride.

However, we expect a different pattern of results for anticipated shame. Anticipated shame is not predicted to be effective at impacting self-control when consumers are in a state of low self-focus because shame focuses attention on the tempting stimulus in this naturally occurring, low self-focus situation. However, if consumers who anticipate shame from consumption are induced to have a high self-focus by being placed in front of a mirror, their attention should shift from the tempting stimulus to the self, enhancing their self-control abilities. Thus, we hypothesize an interaction between anticipated emotions (shame vs. pride) and self-focus (high vs. low) on self-control. Study 2 also examines self- 
control both behaviorally (quantity of cake eaten) and subjectively (self-reported feelings of control over the situation).

\section{Procedure}

One hundred and five respondents (52.4\% male, $45.7 \%$ female (2 respondents did not report gender); ages 18-60; median age=22) participated in this experiment. Respondents included students and staff from a large university. The recruitment method was identical to that reported for Study 1 , and respondents were paid for their participation.

The procedure was identical to that of Study 1 with the exception of the self-focus manipulation. Similar to Study 1, respondents were assigned to different conditions and were presented with a box containing a piece of chocolate cake. The self-focus manipulation was similar to that used by previous research (Beaman et al., 1979; Diener \& Wallbom, 1976). Respondents in the high self-focus condition sat at a desk facing a mirror on a stand. The mirror was angled so that when respondents sat down at the desk their full face (and not the cake) was in view. The mirror was absent for respondents in the low self-focus condition.

After eating the cake, respondents noted how difficult it was for them to control how much cake they ate ( $1=$ not at all, $7=$ =very much). Measures of time of day, extent of hunger, respondents' general liking of chocolate cake, and chronic self-control (Tangney, Baumeister, \& Boone, 2004) were assessed. We found no differences in these variables across the experimental conditions. Nor did the results differ when these variables were included as covariates in the analyses. Thus, these results are not reported.

\section{$\underline{\text { Results }}$}

Significantly more respondents in the anticipated pride/high self-focus condition (57.1\%) chose not to eat the cake at all compared to respondents in the other three experimental conditions $(23.1 \%$ in the anticipated pride/low self-focus condition, $23.1 \%$ in the anticipated shame/high self-focus condition, and $31 \%$ in the anticipated shame/low self-focus condition; all $\left.p^{\prime} s<.05\right)$. These results suggest that when consumers anticipate pride, self-control is enhanced further when the environment fosters a focus on the self. However, the goal of Study 2 is to determine how the self-focus manipulation enhanced the degree of self-control. Therefore a critical analysis concerns how much consumers who chose to consume actually consumed. 


\section{Consumption}

A $2 \times 2$ ANOVA with anticipated emotions and self-focus as the independent variables and the amount consumed as the dependent variable revealed a main effect of self-focus and the hypothesized anticipated emotions and self-focus interaction. Respondents in the high self-focus conditions consumed less than respondents in the low self-focus conditions ( $M=2.46$ vs. $M=3.40, F(1,64)=3.99, p=.05)$. However, this main effect of self-focus was qualified by the hypothesized anticipated emotion×selffocus interaction $(F(1,64)=4.02, p<.05)$. Consistent with our theorizing, consumers in the shame/low self-focus condition ate significantly more cake $(M=4.29)$ than respondents in the shame/high self-focus $(M=2.34)$, pride low self-focus $(M=2.65)$, and the pride/high self-focus $(M=2.66)$ conditions. There were no significant differences between consumers in the latter 3 conditions.

\section{Feelings of Control}

A $2 \times 2$ ANCOVA with anticipated emotions and self-control as the independent variables and subjective self-control difficulty as the dependent variable (using self-reported liking of chocolate cake as a covariate) revealed a main effect for self-focus and the hypothesized anticipated emotions and selffocus interaction. Consumers reported more self-control difficulty in the low vs. the high self-focus condition ( $M=5.38$ vs. $M=4.13, F(1,63)=4.58, p<.05)$. However, this main effect was qualified by the predicted interaction between anticipated emotions and self-focus $(F(1,63)=3.86, p<.05)$. As expected, consumers in the shame/low self-focus condition reported more difficulty in self-control $(M=6.41)$ than did respondents in the other three conditions; shame/high self-focus $(M=4.05)$, pride/low self-focus $(M=4.50)$, and pride/high self-focus $(M=4.25)$. Contrast analysis revealed no significant differences between consumers in the latter 3 conditions.

\section{General Discussion}

Recent discussions of self-control indicate the need for additional research on how the "cool" or "reflective" system of self-control is powered (Strack et al., 2006; Vohs, 2006). It is interesting to consider the role of anticipated emotions in the reflective system since they tap into the "cool" reflective system by virtue of their future focus yet some may still have emotional properties typically linked to the "hot" impulsive system. We propose that anticipated emotions associated with self-control contexts (i.e. shame anticipated from giving into temptation and the pride anticipated from resisting temptation) differ in their activation of the hot and cool systems and do so in terms of the attentional focus they bring to the self-regulatory context. The conclusions obtained from Studies 1 and 2 support 
these predictions using self-reported data (self-reported choice and thought protocols), behavioral data (amount consumed), and observational data (number and size of bites).

Study 1 finds that anticipating pride from non-consumption seems to activate the cool system and enhance self-control, both immediately and over time. Our verbal data suggest that one reason why anticipated shame may be less effective than anticipated pride is that it renders a focus on the tempting stimulus and its consummatory properties. In contrast, anticipated pride seems to shift attention away from the stimulus and its consummatory properties to the self and the goal of self-control. Study 2 tests this idea further, by suggesting that attentional focus is indeed responsible for the differential efficacy of anticipated pride vs. shame. Attentional focus was manipulated to reveal that anticipating shame can induce self-control when consumers are in environments that heighten a focus on the self. A heightened focus on the self does not further strengthen the facilitative effects of anticipated pride. Our results for quantity consumed and feelings of control were identical both in the high and low self-focus anticipated pride conditions. Hence, attention on the self arising from anticipated pride seems to be responsible for the effects we observe. Notably, our results in Study 2 do not suggest that anticipated pride is more effective than anticipated shame, but even anticipated shame can result in enhanced self-control when attention is shifted from the tempting stimulus to the self.

\section{Directions for Future Research}

Future research might investigate boundary conditions for the effects observed here. We hypothesized that anticipated pride enhances self-control given its ability to shift attention from the consummatory or hot properties of the stimulus to the self. As such, factors that impact a consumer's ability to maintain their focus on the self might undermine self-control. Hence, one might surmise that the effects of anticipated pride will be weakened under conditions of distraction where consumers cannot refocus attention on the self. For example, anticipated pride may be weakened by social pressure and a shift of attention from the self to the social group to which one belongs (see Wood and Bettman 2007) or for habitual consumption (Ji \& Wood, 2007). Observing others' self-gratification (vs. self-control) actions may make one believe that yielding to temptation, not resisting it, is the socially appropriate course of action.

Second, while our research has examined the impact of two specific anticipated emotions on self-control, one wonders whether consumers can anticipate various combinations of emotions (see Kramer and Yoon 2007). Several combinations are possible (1) pleasure and pride (approach-approach conflicts), (2) shame and deprivation (avoidance-avoidance conflicts), (3) pleasure and shame, (4) pride 
and deprivation (approach-avoidance conflicts), (5) pleasure and deprivation, and (6) shame and pride (no conflict). One might expect that certain combinations of emotions are easier to anticipate and more naturally occurring than are others. For example, it might be easier to anticipate pleasure and deprivation because both support yielding to temptation. It may be difficult for consumers to anticipate shame from non-consumption and deprivation from consumption because the two emotions imply different behaviors and neither is pleasant to envision. Although prior research has explored the effects of mixed emotions on self-control (Ramanathan \& Williams, 2007), the effects of mixed anticipated emotions remains unexplored.

Finally, in this research we have not exhausted the anticipated emotions that might be relevant to self-control. Future research might examine whether emotions like guilt, regret from consuming, or regret from not consuming-have similar or divergent effects to those observed here. Richard, de Vries, and van der Pligt (1998) found that anticipated regret from having unprotected sex increased the likelihood that they would resist the temptation to engage in unprotected sex. These results suggest a facilitative effect of anticipated regret on self-control. Anticipated regret could be sufficiently broad to include shame. However, it could be a narrower and more specific emotion that encapsulates not selfreproach (as is implied by shame) but action reproach (from specific behaviors). Self-reproach from action is perhaps more similar to the concept of guilt (Tangney \& Dearing, 2002). Interestingly though, while Richard et al.'s (1998) work suggests that anticipated regret can enhance self-control, some recent research suggests the anticipating the guilt from consuming a tempting stimulus undermines selfcontrol-perhaps because of the mental link an individual has established between guilt and pleasure (guilty pleasures) (Chun, Patrick, \& MacInnis, 2006). The effects of anticipated regret, anticipated guilt, and anticipated shame on self-control deserve more attention in future research.

\section{References}

Bagozzi, R. P., Baumgartner, H., \& Pieters, R. (1998). Goal-directed emotions. Cognition and Emotion, 12(1), 1-26.

Baumeister, R. F., Bratslavsky, E., Muraven, M., \& Tice, D. M. (1998). Ego depletion: is the active self a limited resource? Journal of Personality and Social Psychology, 74(5), 1252-1265.

Baumeister, R. F., Vohs, K. D., De Wall, C. N., \& Zhang, L. (2007). How emotion shapes behavior: feedback, anticipation, and reflection, rather than direct causation. Personality and Social Psychology Review, 11(2), 167-203. 
Baumeister, R. F., Sparks, E. A., Stillman, T. F., \& Vohs, K. D. (2008). Free will in consumer behavior: selfcontrol, ego depletion, and choice. Journal of Consumer Psychology, 18(1), 4-13.

Beaman, E. A., Klentz, B., Diener, E., \& Svanum, S. (1979). Self-awareness and transgression in children: two field studies. Journal of Personality and Social Psychology, 37(10), 1835-1846.

Chun, H., Patrick, V. M., \& Maclnnis, D. J. (2006). Making prudent vs. impulsive choices: the role of anticipated shame and guilt on consumer self-control. In G. J. Fitzsimons, \& V. G. Morwitz (Eds.), Advances in Consumer Research, 34, 715-719.

Diener, E., \& Wallbom, M. (1976). Effects of self-awareness on antinormative behavior. Journal of Research in Personality, 10, 111-197.

Fedorikhin, A., \& Patrick, V.M. (2009). What's wrong with having too much fun?: The moderating role of arousal in the influence of positive mood on self-control. Working paper, University of Georgia.

Garg, N., Wansink, B., \& Inman, J. J. (2007). The influence of incidental affect on consumers' food intake. Journal of Marketing, 71(1), 194-206.

Gilbert, D. T., Pinel, E. C., Wilson, T. D., Blumberg, S. J., \& Wheatley, T. P. (1998). Immune neglect: a source of durability bias in affective forecasting. Journal of Personality and Social Psychology, 75(3), 617-638.

Giner-Sorolla, R. (2001). Guilty pleasures and grim necessities: affective attitudes in dilemmas of selfcontrol. Journal of Personality and Social Psychology, 20(2), 206-221.

Heatherton, T. F., Polivy, J., Herman, C. P., \& Baumeister, R. F. (1993). Self-awareness, task failure, and disinhibition: how attentional focus affects eating. Journal of Personality, 71(1), 49-61.

Ji, M. F., \&Wood, W. (2007). Purchase and consumption habits: not necessarily what you intend. Journal of Consumer Psychology, 17(4), 262-276.

Kahneman, D., \& Snell, J. (1992). Predicting a changing taste: do people know what they will like? Journal of Behavioral Decision Making, 5(3), 187-200.

Kramer, T., \& Yoon, S. (2007). Approach-avoidance motivation and the use of affect as information. Journal of Consumer Psychology, 17(2), 128-138.

Lewis, Helen Block (1971). Shame and Guilt in Neurosis. New York: International University.

MacInnis, D. J., \& Patrick, V. M. (2006). Spotlight on affect: affect and affective forecasting in impulse control. Journal of Consumer Psychology, 16(3), 224-231.

MacInnis, D. J., Patrick, V. M., \& Park, C. W. (2005). Looking through the crystal ball: the role of affective forecasting and misforecasting in consumer behavior. Review of Marketing Research, 2, 43-79. 
Mascolo, M. F., \& Fischer, K. W. (1995). Developmental transformations in appraisals for pride, shame, and guilt. In J. P. Tangney, \& K. W. Fischer (Eds.), Self-conscious Emotions: the Psychology of Shame, Guilt, Embarrassment, and Pride (pp. 64-113). New York: Guilford Press.

Metcalfe, J., \& Mischel, W. (1999). A Hot/cool-system analysis of delay of gratification: dynamics of willpower. Psychological Review, 106(1), 3-19.

Mischel, W., \& Baker, N. (1975). Cognitive appraisals and transformations in delay behavior. Journal of Personality and Social Psychology, 31, 254-261.

Mischel, W., Ebbesen, E. B., \& Zeiss, A. R. (1972). Cognitive and attentional mechanisms in delay of gratification. Journal of Personality and Social Psychology, 21, 204-218.

Mukhopadhyay, A., \& Johar, G. V. (2007). Tempted or not? The effect of recent purchase history on responses to affective advertising. Journal of Consumer Research, 33, 445-453.

Mukhopadhyay, A., \& Johar, G. V. (2009). Indulgence as self-reward for prior shopping restraint: a justification-based mechanism. Journal of Consumer Psychology forthcoming. doi:10.1016/j.jcps.2009.02.016.

Ortony, Andrew, Clore, Gerald L., \& Collins, Allan (1988). The Cognitive Structure of Emotions. Cambridge, MA: Cambridge University Press.

Patrick, V. M., Maclnnis, D. J., \& Park, C. W. (2007). Not as happy as I thought I'd be: affective misforecasting and product evaluations. Journal of Consumer Research, 33(4), 479-490.

Ramanathan, S., \& Williams, P. (2007). Immediate and delayed emotional consequences of indulgence: the moderating influence of personality type on mixed emotions. Journal of Consumer Research, $34(2), 212-224$.

Richard, R., de Vries, N., \& Van der Pligt, J. (1998). Anticipated regret and precautionary sexual behavior. Journal of Applied Social Psychology, 28 (15), 1411-1428.

Strack, F., Werth, L., \& Deutsch, R. (2006). Reflective and impulsive determinants of consumer behavior. Journal of Consumer Psychology, 16, 205-216.

Tangney, J. P. (1999). The self-conscious emotions: shame, guilt, embarrassment and pride. In T. Dalgleish, \& M. J. Power (Eds.), Handbook of Cognition and Emotion (pp. 541-568). New York: John Wiley.

Tangney, J. P., \& Dearing, R. L. (2002). Shame and Guilt. New York: Guilford.

Tangney, J. P., Baumeister, R. F., \& Boone, A. L. (2004). High self-control predicts good adjustment, less pathology, better grades, and interpersonal success. Journal of Personality, 72(2), 271-324. 
Tracy, J. L., \& Robins, R. W. (2004). Putting the self into self-conscious emotions: a theoretical model. Psychological Inquiry, 15(2), 103-125.

Tracy, J. L., \& Robins, R.W. (2007). The psychological structure of pride: a tale of two facets. Journal of Personality and Social Psychology, 92(3), 506-525.

Vohs, K. D. (2006). Self-regulatory resources power the reflective system: evidence from five domains. Journal of Consumer Psychology, 16, 217-223.

Wood, S. L., \& Bettman, J. R. (2007). Predicting happiness: how normative feeling rules influence (and even reverse) durability bias. Journal of Consumer Psychology, 17(3), 188-201. 